\title{
Uterine stump leiomyosarcoma after sub-total hysterectomy: a case report
}

\begin{abstract}
Introduction: Uterine sarcomas considered as one of the aggressive tumors of uterine malignancies. It is one of the mesenchymal tumors that originate from smooth muscle of the uterus which is a rare tumor that accounts for $2 \%$ to $5 \%$ of all uterine malignancies. Very few cases are reported in the literature. Our patient has a unique history of PelvicAbdominal swelling reaching xiphisternum level after subtotal hysterectomy one year ago. Abnormal genital bleeding not responding to medical treatment is the usual presentation in uterine sarcoma like in our patient. We report an original case report of an abnormal sequence of this rare tumor arising from the uterine stump after subtotal hysterectomy.
\end{abstract}

Case presentation: A 42-year-old nulliparous woman presented to our gyne-oncology unit in El-Galaa Maternity Teaching Hospital in June 2020 with a significant rapid increase in abdominal circumference, symptoms caused by abdominal pressure (vomiting and constipation) and abnormal genital bleeding after laparotomic sub-total hysterectomy one year ago. Tumor marker CA-125 was raised, LDH was raised and a MRI scan showed a huge mass arising from the pelvis. An exploratory laparotomy was performed and the histopathology report confirmed the diagnosis of uterine leiomyosarcoma weighing around $22 \mathrm{~kg}$.

Conclusion: Because of their rarity, uterine sarcomas are not suitable for screening. Diagnosis by histopathologic examination and surgery is the only treatment. Pre-operative MRI with contrast for abdomen and pelvis is highly recommended to exclude abdomen metastatic sarcoma if the tumor is confined to the pelvis only.
Volume 12 Issue 4 - 202I

\section{Mohamed Ayaty,' 'Mohamed Abdallah, ${ }^{2}$ Ahmed Elghandor,' Haitham Abdel Wahab ${ }^{2}$ 'Gyne-oncology Department, National Cancer Institute, Egypt ${ }^{2}$ Gyne-oncology Department, El-Galaa Maternity Teaching Hospital, Egypt}

Correspondence: Haitham Abdel Wahab, Gyne-oncology Department, El-Galaa Maternity Teaching Hospital, Egypt, Emaildr_haytham89@yahoo.com

Received: July 02, 202I | Published: August 04, 2021

Keywords: a rare case, uterine stump leiomyosarcoma

\section{Introduction}

Uterine sarcomas considered as one of the aggressive tumors of uterine malignancies. It is one of the mesenchymal tumors that originates from smooth muscle of the uterus which is a rare tumor that accounts for $2 \%$ to $5 \%$ of all uterine malignancies. ${ }^{1}$

The most common type of uterine sarcomas is Leiomyosarcomas (LMSs), with an annual incidence of $2-7 / 100,000$ per women, followed by endometrial stromal sarcomas with an annual incidence of $1-2 /$ million per women. ${ }^{2}$

\section{Case report}

A female patient 42-years-old admitted to our Gyne-Oncology unit in El-Galaa Maternity Teaching Hospital, in June 2020 with a significant rapid increase in abdominal circumference, complaining of abdominal pressure symptoms like (constipation and vomiting) and abnormal genital bleeding.

One year earlier, at another hospital she had subtotal hysterectomy for a suspected uterine myoma measured $10 \times 13 \times 9 \mathrm{~cm}$, which was histologically confirmed a leiomyoma (Figure 1).

General examination, pallor was present HGB was $6.9 \mathrm{gm}$, and the patient's vital signs were normal. She was faty built.

Abdominal examination, a lobulated midline masses occupying the whole abdomen and pelvis. The upper border reached xiphisternum level. The lower border could not be felt. The masses were firm to hard in consistency with restricted mobility.

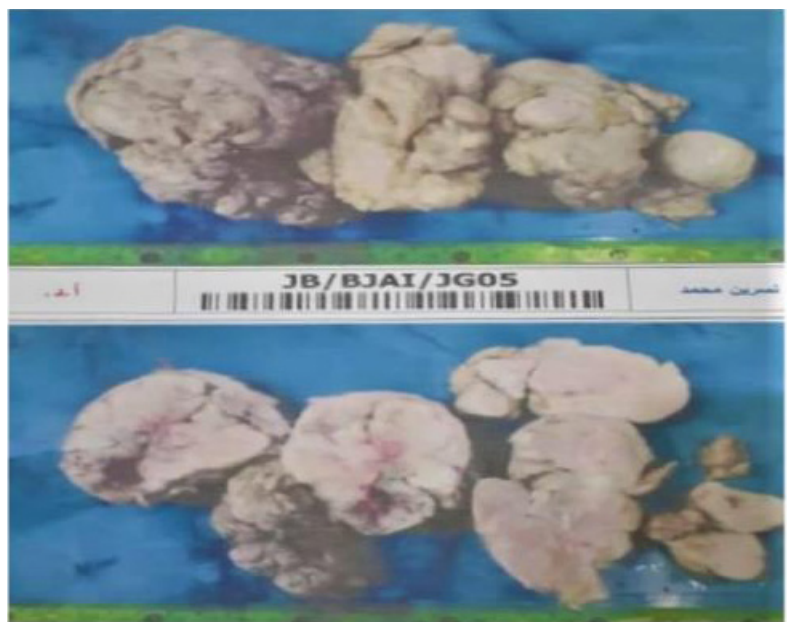

Figure I Gross picture of the previous leiomyoma of subtotal hysterectomy.

Vaginal examination, the cervix was normal, and there was blood coming through cervix.

Investigations, Magnetic resonance Imaging (MRI) scan findings were suggestive of a large Lobulated masses compressing intestinal loops , arising from the uterine stump and extending into the abdomen, measuring $30 \times 24 \times 13 \mathrm{~cm}$, adherent to both bowel and bladder (Figure 2) (Figure 3). CA125 was $132.3 \mathrm{ng} / \mathrm{mL}(0-35 \mathrm{ng} / \mathrm{mL})$, LDH was 1127 $(140-280 \mathrm{U} / \mathrm{L})$, the remain results were normal, and the patient was prepared for Mid-line exploration. 


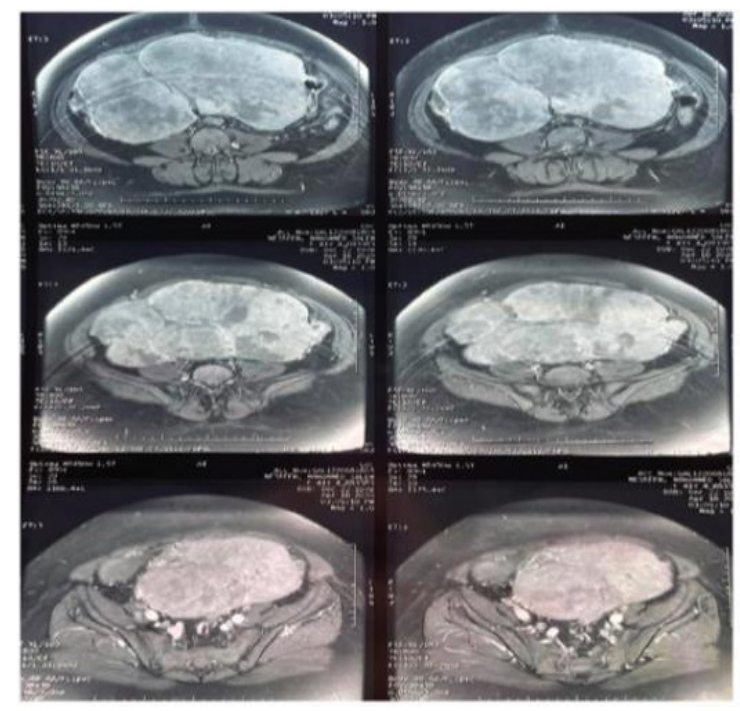

Figure 2 MRI of the Tumor before surgery.

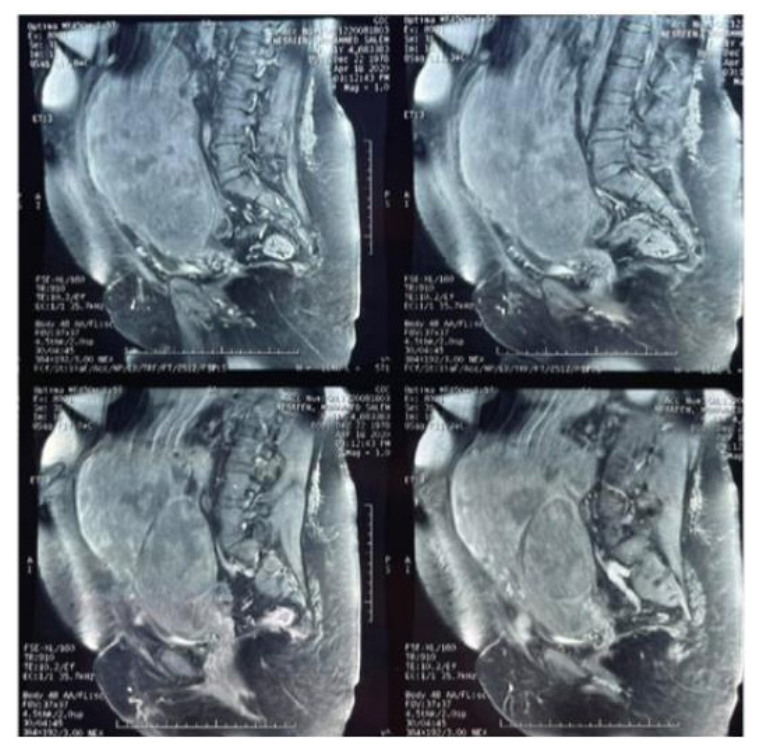

Figure $3 \mathrm{MRI}$ of the Tumor before surgery.

Intra-operatively, through Mid-line exploration, the abdominpelvic cavity was completely obliterated with huge lobulated highly vascular masses (Figure 4) (Figure 5).

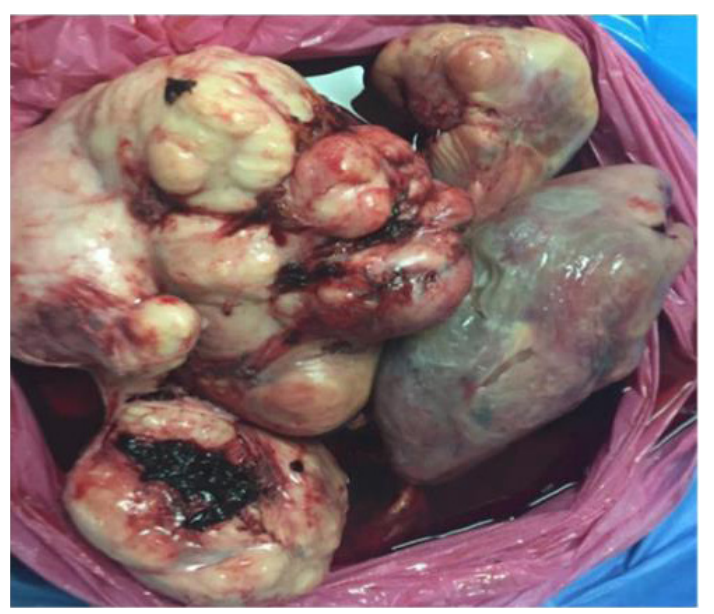

Figure 4 The whole tumor lobules weighing around $22 \mathrm{~kg}$.

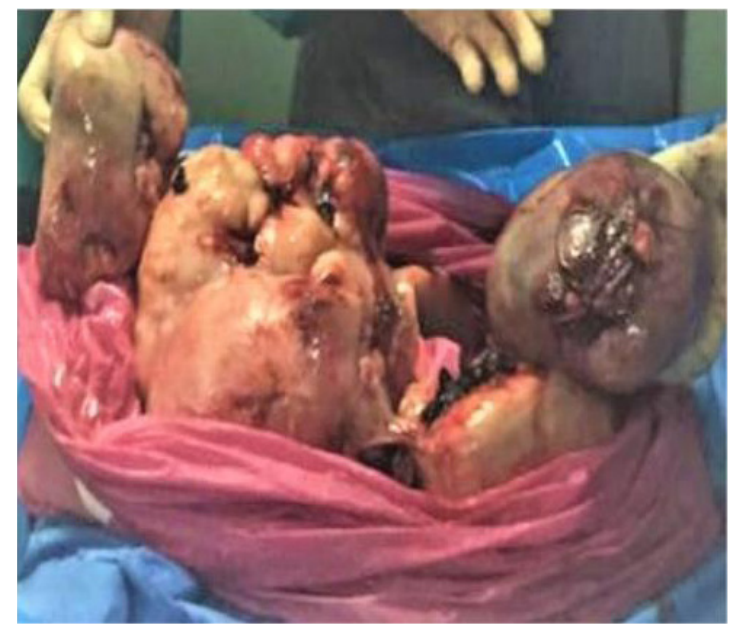

Figure $\mathbf{5}$ The whole tumor.

The masses were adhered to the omentum, several intestinal loops, Also there was evidence of infiltration in a small site in transverse colon and bladder. Total removal of uterine stump with safety margin from vagina, and the whole lobulated masses, excision of infiltrated site in transverse colon with safety margin and primary repair was done without covering ileostomy, partial bladder cystectomy due to infiltration with safety margin was preserved, salpingo-oophorectomy bilateral was done, infra-colic omentectomy, and appendectomy.

Histological examination showed Active spindle cell tumor with cellular atypia, Focally Necrotized, consistent with low grade Leiomyosarcoma (Figure 6). Gross pathology, Multiple huge defined lobulated firm grayish masses weighing around $22 \mathrm{~kg}$, measured $32 \times 23 \times 14 \mathrm{~cm} \backslash 25 \times 17 \times 7 \mathrm{~cm} \backslash 17 \times 9.5 \times 10 \mathrm{~cm}$.

After 10 days in the ward, the patient was discharged in good condition.

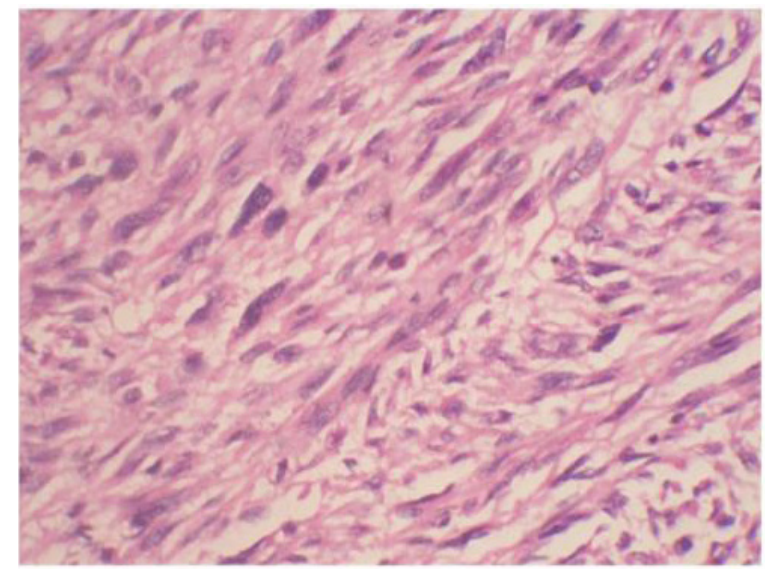

Figure 6 Microscopic examination showing cellular tumor arranged in bundle of spindle cells with hyperchromatic nuclei.

\section{Discussion}

Based on statistics, Any patient has an bulky uterus due to uterine fibroid exceeding $7 \mathrm{~cm}$ is at risk to have a pathology of a malignant uterine smooth muscle tumor. Leiomyosarcoma is rare which the main pathology of the cases arise de novo rather than from the malignant transformation of benign myomas. ${ }^{3}$

The medical history findings like parity, time of menarche and menopause considered to be risk factors are inconclusive. The long 
term use of Tamoxifen in women with breast cancer associate with an increase in the risk of uterine sarcomas three times. ${ }^{4}$

In 2014, the FDA launches a global warning against the widespread of laparoscopic morcellations to deal with fibroids, Due to the late diagnosis of uterine occult malignant smooth muscle tumors like uterine sarcomas and their potential dissemination in the abdominal cavity.

Due to uterine sarcomas rarity, The existing in literature on the topic remains scarce and, therefore, a management plan including diagnosis, investigations, staging, the proper treatment and followup have to be reached. ${ }^{5}$ The incidence of sarcoma is $1 \%$ to $2 \%$ in postmenopausal women. The main complain of this patients is abnormal uterine bleeding. ${ }^{6}$

As in our patient presented with abnormal genital bleeding and rapidly growing tumor. After one year of laparotomy sub-total hysterectomy.

\section{Conclusion}

Till now, the preferred treatment of uterine sarcomas is surgery as it is difficult for screening. The less the mitotic index in pathology the better the prognosis will be for women with uterine sarcoma.?

The best management will be achieve through multidisciplinary team including a gynecologist, pathologist, oncologist and radiologist by setting the management plan of treatment, especially in young patients seeking fertility.

Pre-operative MRI with contrast for abdomen and pelvis is highly recommended to exclude abdomen metastatic sarcoma if the tumor is confined to the pelvis only.

\section{Acknowledgments}

The authors would like to thank Dr. Ebtihag Shafik (Head of Obstetrics and Gynecology Department in El-Galaa Maternity Teaching Hospital) for her appreciated efforts in this case.

The authors would like to thank Dr. Wael Fathy (Head of Anaesthesia Department in El-Galaa Maternity Teaching Hospital) for his appreciated efforts in this case.

\section{Funding}

None.

\section{Consent}

Written informed consent was obtained from the patient for publication of this case report. A copy of the written consent is available for review by the Editor-in-Chief of this journal.

\section{Authors' contributions}

All authors have read and approved the final manuscript.

\section{Conflicts of interest}

The authors declare that they have no competing interests.

\section{References}

1. Forney JP, Buschbaum HJ. Classifying staging and treating uterine sarcomas. Contemporary Ob Gyn. 1981;18(3):47.

2. K Pietzner, N Buttmann-Schweiger, J Sehouli, et al. Incidence patterns and survival of gynecological sarcoma in Germany. Int J Gynecol Cancer. 2018;28:134-138

3. Schwartz PE, Kelly MG. Malignant transformation of myomas: myth or reality? Obstet. Gynecol. Clin North Am. 2006;33:183-198.

4. Bergman L, Beelen ML, Gallee MP, et al. Risk and prognosis of endometrial cancer after tamoxifen for breast cancer. Lancet. 2000;356:881-887.

5. Dall'Asta A, Gizzo S, Musarò A, et al. Uterine smooth muscle tumors of uncertain malignant potential (STUMP): pathology, follow-up and recurrence. Int J Clin Exp Pathol. 2014;7:8136-8142.

6. Wickerham DL, Fisher B, Wolmark N, et al. Association of tamoxifen and uterine sarcoma. J Clin Oncol. 2002;20:2758-2760.

7. Major FJ, Blessing JA, Silverberg SG, et al. Prognostic factors in early stage uterine sarcoma. A Gynaecologic Oncology group study. Cancer. 1993;71(4):1702-1709. 